\title{
Mesomelia-synostoses syndrome
}

INSERM

\section{Source}

INSERM. (1999). Orphanet: an online rare disease and orphan drug data base.

Mesomelia-synostoses syndrome. ORPHA:2496

Mesomelia-Synostoses syndrome (MSS) is a syndromal osteochondrodysplasia due to a contiguous gene deletion syndrome, characterized by progressive bowing of forearms and forelegs leading to mesomelia, progressive intracarpal or intratarsal bone fusion and fusion of metacarpal bones with proximal phalanges, ptosis, hypertelorism, abnormal soft palate, congenital heart defect, and ureteral anomalies. 